\title{
Existence of Solutions to a Class of Semilinear Elliptic Problem with Nonlinear Singular Terms and Variable Exponent
}

\author{
Ying Chu, ${ }^{1}$ Yanchao Gao, ${ }^{1}$ and Wenjie Gao ${ }^{2}$ \\ ${ }^{1}$ School of Science, Changchun University of Science and Technology, Changchun 130022, China \\ ${ }^{2}$ Institute of Mathematics, Jilin University, Changchun 130012, China \\ Correspondence should be addressed to Yanchao Gao; ychaogao@163.com
}

Received 1 April 2016; Accepted 12 May 2016

Academic Editor: Maria Alessandra Ragusa

Copyright (c) 2016 Ying Chu et al. This is an open access article distributed under the Creative Commons Attribution License, which permits unrestricted use, distribution, and reproduction in any medium, provided the original work is properly cited.

The authors of this paper prove the existence and regularity results for the homogeneous Dirichlet boundary value problem to the equation $-\operatorname{div}\left(M(x) \nabla u_{n}\right)=f(x) / u^{\alpha(x)}$ with $f \in L^{m}(\Omega)(m \geqslant 1)$ and $\alpha(x)>0$. The results show the dependence of the summability of $f$ in some Lebesgue spaces and on the values of $\alpha(x)$.

\section{Introduction}

In this paper, we study the existence of solutions for the following semilinear elliptic problem with nonlinear singular terms and variable exponent:

$$
\begin{aligned}
-\operatorname{div}(M(x) \nabla u) & =\frac{f(x)}{u^{\alpha(x)}}, \quad x \in \Omega, \\
u & =0, \quad x \in \partial \Omega,
\end{aligned}
$$

where $\Omega$ is a bounded domain in $R^{N}(N \geqslant 2)$ with smooth boundary $\partial \Omega, \alpha(x)$ is a continuous function on $\bar{\Omega}, \alpha(x)>$ $0, \alpha^{+}=\sup _{x \in \bar{\Omega}} \alpha(x), \alpha^{-}=\inf _{x \in \bar{\Omega}} \alpha(x), f$ is a nonnegative function belonging to the Lebesgue space $L^{m}(\Omega)$, for some suitable $m \geqslant 1$, and $M$ is a bounded positive definite matrix; that is, there exist $0<\gamma \leqslant \beta$ such that

$$
\begin{aligned}
\gamma|\eta|^{2} & \leqslant(M(x) \eta) \cdot \eta, \\
|M(x)| & \leqslant \beta,
\end{aligned}
$$

for every $\eta$ in $R^{N}$, for almost every $x$ in $\Omega$.

Problem (1) has been widely applied in many areas, such as the contexts of chemical heterogeneous catalysts, nonNewtonian fluids, and also the theory of heat conduction in electrically conducting materials; see [1-4] for detailed discussion.
Problem (1) has been extensively studied in the past. In [5], Lazer and Mckenna dealt with model (1) with $f$, a continuous function; they proved that the solution was in $H_{0}^{1}(\Omega)$ if and only if $\alpha<3$, while it was not in $C^{1}(\bar{\Omega})$ if $\alpha>1$.

Later, Lair and Shaker in [6] studied the existence of solutions to the elliptic equation

$$
\begin{aligned}
-\Delta u & =f(x) u^{-\alpha}, \quad x \in \Omega, \\
u & =0, \quad x \in \partial \Omega .
\end{aligned}
$$

They proved that problem (3) with $0<\alpha<1$ has a unique weak positive solution in $H_{0}^{1}(\Omega)$ if $f(x)$ is a nonnegative nontrivial function in $L^{2}(\Omega)$.

Moreover, the results of Lair and Shaker were generalized by Shi and Yao (see [7]); they studied the following problem:

$$
\begin{gathered}
-\Delta u=f(x, u), \quad x \in \Omega, \\
u=\varphi, \quad x \in \partial \Omega,
\end{gathered}
$$

where $\Omega$ is a bounded domain in $R^{N}, N \geqslant 2, \varphi \geqslant 0$ may take the value 0 on $\partial \Omega$, and $f(x, s)$ is possibly singular near $s=$ 0 . They proved the existence and the uniqueness of positive solutions without assuming monotonicity or strict positivity on $f(x, s)$. 
Recently, Boccardo and Orsina in [8] studied the existence, regularity, and nonexistence of solutions for the following problem:

$$
\begin{aligned}
-\operatorname{div}(M(x) \nabla u) & =\frac{f(x)}{u^{\alpha}}, \quad x \in \Omega, \\
u & =0, \quad x \in \partial \Omega .
\end{aligned}
$$

They discussed the dependence of the results on the summability of $f$ and the values of $\alpha$. For the other results of singular elliptic equations, see $[9,10]$. In this paper, we generalize the results in [8] to the case when $\alpha$ is a variable exponent by applying the method of regularization, Schauder fixed point theorem, the integrability of solution to the approximate problem with $n=1$, and a necessary compactness argument to overcome some difficulties arising from the singular terms with variable exponent.

\section{Preliminaries}

Firstly, we give the definition of weak solutions to problem (1).

Definition 1. A function $u \in H_{0}^{1}(\Omega)$ is called a weak solution of problem (1), if the following identity holds:

$$
\int_{\Omega} M(x) \nabla u \nabla \varphi d x=\int_{\Omega} \frac{f}{u^{\alpha(x)}} \varphi d x, \quad \forall \varphi \in C_{0}^{1}(\Omega) .
$$

In order to prove our results, we will consider the following approximation problem:

$$
\begin{aligned}
-\operatorname{div}\left(M(x) \nabla u_{n}\right) & =\frac{f_{n}}{\left(u_{n}+1 / n\right)^{\alpha(x)}}, \quad x \in \Omega, \\
u_{n} & =0, \quad x \in \partial \Omega,
\end{aligned}
$$

where $n \in N, f_{n}(x)=\min \{f(x), n\}$.

Lemma 2. Problem (7) has a nonnegative solution $u_{n}$ in $H_{0}^{1}(\Omega) \bigcap L^{\infty}(\Omega)$.

Proof. Let $n \in N$ be fixed and $\omega$ a function in $L^{2}(\Omega)$. It is not difficult to prove that the following problem has a unique solution $v \in H_{0}^{1}(\Omega) \bigcap L^{\infty}(\Omega)($ see $[11,12])$ :

$$
\begin{aligned}
-\operatorname{div}(M(x) \nabla v) & =\frac{f_{n}}{(|\omega|+1 / n)^{\alpha(x)}}, \quad x \in \Omega, \\
v & =0, \quad x \in \partial \Omega .
\end{aligned}
$$

So, for any $\omega \in L^{2}(\Omega)$, we define the mapping $\Gamma: L^{2}(\Omega) \rightarrow$ $H_{0}^{1}(\Omega) \bigcap L^{\infty}(\Omega)$ as $\Gamma(\omega)=v$. Taking $v$ as a test function, we have, using (2),

$$
\begin{aligned}
\gamma \int_{\Omega}|\nabla v|^{2} d x & \leqslant \int_{\Omega}(M(x) \nabla v) \cdot \nabla v d x \\
& =\int_{\Omega} \frac{f_{n} v}{(|\omega|+1 / n)^{\alpha(x)}} d x \\
& \leqslant n^{1+\alpha^{+}} \int_{\Omega}|v| d x .
\end{aligned}
$$

By Poincaré inequality (on the left hand side) and Hölder's inequality (on the right hand side), we get that

$$
\int_{\Omega}|v|^{2} d x \leqslant C n^{1+\alpha^{+}}\left(\int_{\Omega}|v|^{2} d x\right)^{1 / 2},
$$

for some constant $C$ independent of $\omega$. This implies that

$$
\|v\|_{L^{2}(\Omega)} \leqslant C n^{1+\alpha^{+}}
$$

Therefore, the ball of $L^{2}(\Omega)$ of radius $C n^{1+\alpha^{+}}$is invariant under the mapping $\Gamma$. Since the embedding $H_{0}^{1}(\Omega) \hookrightarrow L^{2}(\Omega)$ is compact, we obtain that $\Gamma$ is a compact operator and $\|v\|_{L^{2}(\Omega)} \leqslant C$. It is also easy to prove that $\Gamma$ is continuous on $L^{2}(\Omega)$, so by Schauder's fixed point theorem, we get that there exists a function $u_{n} \in H_{0}^{1}(\Omega)$, for every fixed $n \in N$, such that $u_{n}=S\left(u_{n}\right)$; that is, problem (7) has a solution. Since $f_{n} /\left(u_{n}+1 / n\right)^{\alpha(x)} \geqslant 0$, the maximum principle implies that $u_{n} \geqslant 0$. Since the right hand side of (7) belongs to $L^{\infty}(\Omega)$, the result of Theorem 4.2 in [13] implies that $u_{n} \in L^{\infty}(\Omega)$.

Lemma 3. The sequence $u_{n}$ is increasing with respect to $n$, $u_{n}>0$ in $\Omega$, and for every $\Omega^{\prime} \subset \subset \Omega$ there exists $C_{\Omega^{\prime}}>0$ (independent of $n$ ) such that

$$
u_{n}(x) \geqslant C_{\Omega^{\prime}}>0
$$

$$
\text { for every } x \in \Omega^{\prime} \text {, for every } n \in N \text {. }
$$

Proof. Due to $0 \leqslant f_{n} \leqslant f_{n+1}$ and $\alpha(x)>0$, we have that

$$
\begin{aligned}
-\operatorname{div}\left(M(x) \nabla u_{n}\right) & =\frac{f_{n}}{\left(u_{n}+1 / n\right)^{\alpha(x)}} \\
& \leqslant \frac{f_{n+1}}{\left(u_{n}+1 /(n+1)\right)^{\alpha(x)}}, \\
-\operatorname{div}\left(M(x) \nabla u_{n+1}\right) & =\frac{f_{n+1}}{\left(u_{n+1}+1 /(n+1)\right)^{\alpha(x)}},
\end{aligned}
$$

so that

$$
\begin{aligned}
- & \operatorname{div}\left(M(x) \nabla\left(u_{n}-u_{n+1}\right)\right) \\
\leqslant & f_{n+1}\left(\frac{1}{\left(u_{n}+1 /(n+1)\right)^{\alpha(x)}}\right. \\
- & \left.\frac{1}{\left(u_{n+1}+1 /(n+1)\right)^{\alpha(x)}}\right)=f_{n+1} \\
& \cdot \frac{\left(u_{n+1}+1 /(n+1)\right)^{\alpha(x)}-\left(u_{n}+1 /(n+1)\right)^{\alpha(x)}}{\left(u_{n}+1 /(n+1)\right)^{\alpha(x)}\left(u_{n+1}+1 /(n+1)\right)^{\alpha(x)}} .
\end{aligned}
$$

Choosing $\left(u_{n}-u_{n+1}\right)_{+}=\max \left\{u_{n}-u_{n+1}, 0\right\}$ as a test function, observing that

$$
\begin{aligned}
& \left(\left(u_{n+1}+\frac{1}{n+1}\right)^{\alpha(x)}-\left(u_{n}+\frac{1}{n+1}\right)^{\alpha(x)}\right) \\
& \cdot\left(u_{n}-u_{n+1}\right)_{+} \leqslant 0,
\end{aligned}
$$


and applying (2), we get that

$$
0 \leqslant \gamma \int_{\Omega}\left|\nabla\left(u_{n}-u_{n+1}\right)_{+}\right|^{2} d x \leqslant 0
$$

This implies that $\left(u_{n}-u_{n+1}\right)_{+}=0$ a.e. in $\Omega$; that is, $u_{n} \leqslant$ $u_{n+1}$ for every $n \in N$. Since the sequence $\left\{u_{n}\right\}$ is increasing with respect to $n$, we only need to prove that (12) holds for $u_{1}$. Applying Lemma 2, we know that $u_{1} \in L^{\infty}(\Omega)$; that is, there exists a constant $C$ (depending only on $\Omega$ and $N$ ) such that

$$
\left\|u_{1}\right\|_{L^{\infty}(\Omega)} \leqslant C\left\|f_{1}\right\|_{L^{\infty}(\Omega)} \leqslant C
$$

and then

$$
-\operatorname{div}\left(M(x) \nabla u_{1}\right)=\frac{f_{1}}{\left(u_{1}+1\right)^{\alpha(x)}} \geqslant \frac{f_{1}}{(C+1)^{\alpha(x)}} .
$$

Due to $f_{1} /(C+1)^{\alpha(x)} \geqslant 0, f_{1} /(C+1)^{\alpha(x)} \not \equiv 0$, the strong maximum principle implies that $u_{1}>0$ in $\Omega$ and (12) holds for $u_{1}$. The monotonicity of $u_{n}$ implies that (12) holds for $u_{n}$.

Remark 4. If $u_{n}$ and $v_{n}$ are two solutions of (7), following the lines of the proof of the first part in Lemma 3 we may show that $u_{n} \leqslant v_{n}$. By symmetry, this implies that the solution of (7) is unique.

Lemma 5. The solution $u_{1}$ to problem (7) with $n=1$ satisfies

$$
\int_{\Omega} u_{1}^{-r} d x<\infty, \quad \forall r<1
$$

Proof. By $\min \{f(x), 1\} /\left(u_{1}+1\right)^{\alpha(x)} \leqslant 1$ and Lemma 2.2 in [14], we know that there exists $0<\beta<1$ such that $u_{1} \in$ $C^{1, \beta}(\bar{\Omega})$ and $\left\|u_{1}\right\|_{C^{1, \beta}} \leqslant C$, which implies that the gradient of $u_{1}$ exists everywhere; then Hopf Lemma in [15] shows that $\partial u_{1}(x) / \partial v>0$ in $\bar{\Omega}$, where $v$ is the outward unit normal vector of $\partial \Omega$ at $x$. Moreover, following the lines of the proof of Lemma in [5], we get that

$$
\int_{\Omega} u_{1}^{r} d x<\infty, \quad \text { iff } r>-1
$$

We know clearly that the estimates on $u_{n}$ depend on $f$ and $\alpha(x)$, and we will discuss it in different cases.

\section{The Case $0<\alpha^{-} \leqslant \alpha(x) \leqslant \alpha^{+}<1$}

In this case, we obtain a priori estimates on $u_{n}$ in $H_{0}^{1}(\Omega)$ only if $f$ is more regular than $L^{1}(\Omega)$, and we have the following results.

Lemma 6. Let $u_{n}$ be the solution of (7) with $0<\alpha^{-} \leqslant \alpha(x) \leqslant$ $\alpha^{+}<1$, and suppose that $f \in L^{m}(\Omega)$ with $m=2 N /(N+2+$ $\left.(N-2) \alpha^{-}\right)=\left(2^{*} /\left(1-\alpha^{-}\right)\right)^{\prime}$. Then the sequence $\left\{u_{n}\right\}$ is bounded in $H_{0}^{1}(\Omega)$.
Proof. Choosing $u_{n}$ as a test function in (7), by Hölder's inequality, (2), and the fact that $f_{n} \leqslant f$, we get

$$
\begin{aligned}
& \gamma \int_{\Omega}\left|\nabla u_{n}\right|^{2} d x \leqslant \int_{\Omega} \frac{f_{n} u_{n}}{\left(u_{n}+1 / n\right)^{\alpha(x)}} d x \\
& \leqslant \int_{\Omega} f u_{n}^{1-\alpha(x)} d x \leqslant \int_{\Omega} f u_{n}^{1-\alpha^{-}} d x+\int_{\Omega} f u_{n}^{1-\alpha^{+}} d x \\
& \leqslant\|f\|_{L^{m}(\Omega)}\left(\int_{\Omega} u_{n}^{\left(1-\alpha^{-}\right) m^{\prime}} d x\right)^{1 / m^{\prime}}+\|f\|_{L^{m}(\Omega)} \\
& \cdot\left(\int_{\Omega} u_{n}^{\left(1-\alpha^{+}\right) m^{\prime}} d x\right)^{1 / m^{\prime}} \leqslant\|f\|_{L^{m}(\Omega)} \\
& \cdot\left(\int_{\Omega} u_{n}^{\left(1-\alpha^{-}\right) m^{\prime}} d x\right)^{1 / m^{\prime}}+\|f\|_{L^{m}(\Omega)} \\
& \cdot\left(\left(\int_{\Omega} 1^{\left(1-\alpha^{-}\right) /\left(\alpha^{+}-\alpha^{-}\right)} d x\right)^{\left(\alpha^{+}-\alpha^{-}\right) /\left(1-\alpha^{-}\right)}\right. \\
& \left.\cdot\left(\int_{\Omega} u_{n}^{\left(1-\alpha^{-}\right) m^{\prime}} d x\right)^{\left(1-\alpha^{+}\right) /\left(1-\alpha^{-}\right)}\right)^{1 / m^{\prime}}=\|f\|_{L^{m}(\Omega)} \\
& \cdot\left(\int_{\Omega} u_{n}^{\left(1-\alpha^{-}\right) m^{\prime}} d x\right)^{1 / m^{\prime}}+|\Omega|^{\left(\alpha^{+}-\alpha^{-}\right) /\left(1-\alpha^{-}\right) m^{\prime}} \\
& \cdot\|f\|_{L^{m}(\Omega)}\left(\int_{\Omega} u_{n}^{\left(1-\alpha^{-}\right) m^{\prime}} d x\right)^{\left(1-\alpha^{+}\right) /\left(1-\alpha^{-}\right) m^{\prime}} .
\end{aligned}
$$

By the assumption of $m$, we have $\left(1-\alpha^{-}\right) m^{\prime}=2^{*}$, and using Sobolev Embedding Theorem (on the left hand side), we have that

$$
\begin{gathered}
\gamma S\left(\int_{\Omega} u_{n}^{2^{*}} d x\right)^{2 / 2^{*}} \leqslant \gamma \int_{\Omega}\left|\nabla u_{n}\right|^{2} d x \leqslant\|f\|_{L^{m}(\Omega)} \\
\cdot\left(\int_{\Omega} u_{n}^{2^{*}} d x\right)^{1 / m^{\prime}}+|\Omega|^{\left(\alpha^{+}-\alpha^{-}\right) /\left(1-\alpha^{-}\right) m^{\prime}}\|f\|_{L^{m}(\Omega)} \\
\cdot\left(\int_{\Omega} u_{n}^{2^{*}} d x\right)^{\left(1-\alpha^{+}\right) /\left(1-\alpha^{-}\right) m^{\prime}}
\end{gathered}
$$

that is,

$$
\begin{aligned}
& \gamma S\left(\int_{\Omega} u_{n}^{2^{*}} d x\right)^{2 / 2^{*}} \leqslant\|f\|_{L^{m}(\Omega)}\left(\int_{\Omega} u_{n}^{2^{*}} d x\right)^{\left(1-\alpha^{-}\right) / 2^{*}} \\
& \quad+|\Omega|^{\left(\alpha^{+}-\alpha^{-}\right) /\left(1-\alpha^{-}\right) m^{\prime}}\|f\|_{L^{m}(\Omega)}\left(\int_{\Omega} u_{n}^{2^{*}} d x\right)^{\left(1-\alpha^{+}\right) / 2^{*}} .
\end{aligned}
$$

Since $1-\alpha^{+}<1-\alpha^{-}<2$, (22) yields the boundedness of $u_{n}$ in $L^{2^{*}}(\Omega)$. By this estimate and (22), the conclusion follows.

Once we have the boundedness of $u_{n}$, we can prove an existence result for (1).

Theorem 7. Suppose that $f$ is a nonnegative function in $L^{m}(\Omega)(f \not \equiv 0)$, with $m=2 N /\left(N+2+(N-2) \alpha^{-}\right)=$ $\left(2^{*} /\left(1-\alpha^{-}\right)\right)^{\prime}$, and let $0<\alpha^{-} \leqslant \alpha(x) \leqslant \alpha^{+}<1$. Then problem (1) has a solution $u \in H_{0}^{1}(\Omega)$ satisfying (6). 
Proof. Since $u_{n}$ is bounded in $H_{0}^{1}(\Omega)$ by Lemma 6 and $u_{n}$ converges to $u$ pointwise in $\Omega$ (by Lemma 3 ), then we know that there exists $u \in H_{0}^{1}(\Omega)$ such that

$$
\begin{aligned}
& u_{n} \rightarrow u \\
& \quad \text { weakly in } H_{0}^{1}(\Omega) \text { and strongly in } L^{2}(\Omega), \\
& u_{n} \longrightarrow u \quad \text { a.e. in } \Omega, \\
& \nabla u_{n} \rightarrow \nabla u \quad \text { weakly in } L^{2}(\Omega) .
\end{aligned}
$$

So we have that

$$
\begin{aligned}
& \lim _{n \rightarrow \infty} \int_{\Omega}\left(M(x) \nabla u_{n}\right) \cdot \nabla \varphi d x \\
& \quad=\int_{\Omega}(M(x) \nabla u) \cdot \nabla \varphi d x, \quad \forall \varphi \in C_{0}^{1}(\Omega) .
\end{aligned}
$$

Since $u_{n}$ satisfies (12), we get that

$$
0 \leqslant\left|\frac{f_{n} \varphi}{\left(u_{n}+1 / n\right)^{\alpha(x)}}\right| \leqslant \frac{\|\varphi\|_{L^{\infty}(\Omega)} f}{\min \left\{C_{\Omega^{\prime}}^{\alpha^{-}}, C_{\Omega^{\prime}}^{\alpha^{+}}\right\}},
$$

where $\Omega^{\prime}=\{x: \varphi \neq 0\}$. Then by Lebesgue Dominated Convergence Theorem, we have that

$$
\lim _{n \rightarrow \infty} \int_{\Omega} \frac{f_{n} \varphi}{\left(u_{n}+1 / n\right)^{\alpha(x)}} d x=\int_{\Omega} \frac{f \varphi}{u^{\alpha(x)}} d x
$$

Since $u_{n}$ is a solution of (7), this implies that

$$
\int_{\Omega}\left(M(x) \nabla u_{n}\right) \nabla \varphi d x=\int_{\Omega} \frac{f_{n} \varphi}{\left(u_{n}+1 / n\right)^{\alpha(x)}} d x,
$$

$$
\forall \varphi \in C_{0}^{1}(\Omega) .
$$

Letting $n \rightarrow \infty$, combining (25) with (27), we get that

$$
\int_{\Omega}(M(x) \nabla u) \cdot \nabla \varphi d x=\int_{\Omega} \frac{f \varphi}{u^{\alpha(x)}} d x,
$$

which proves that (1) has a solution $u$ in $H_{0}^{1}(\Omega)$.

The summability of $u$ depends on the summability of $f$, which is proved in the next Lemma.

Lemma 8. Suppose that $f \in L^{m}(\Omega), m \geqslant 2 N /(N+2+(N-$ 2) $\alpha^{-}$), and let $0<\alpha^{-} \leqslant \alpha(x) \leqslant \alpha^{+}<1$. Then the solution $u$ of (1) given by Theorem 7 is such that

(i) if $m>N / 2$, then $u \in L^{\infty}(\Omega)$;

(ii) if $2 N /\left(N+2+(N-2) \alpha^{-}\right) \leqslant m<N / 2$, then $u \in L^{s}(\Omega)$, $s=N m\left(1+\alpha^{-}\right) /(N-2 m)$.
Proof. To prove (i), let $k>1$ and define $G_{k}(s)=(s-k)_{+}$. Taking $G_{k}\left(u_{n}\right)$ as a test function in (7), using (2), we get

$$
\begin{aligned}
& \gamma \int_{\Omega}\left|\nabla G_{k}\left(u_{n}\right)\right|^{2} d x \\
& \quad \leqslant \int_{\Omega}\left(M(x) \nabla G_{k}\left(u_{n}\right)\right) \cdot \nabla G_{k}\left(u_{n}\right) d x \\
& \quad=\int_{\Omega} \frac{f_{n} G_{k}\left(u_{n}\right)}{\left(u_{n}+1 / n\right)^{\alpha(x)}} d x .
\end{aligned}
$$

Since $G_{k}\left(u_{n}\right) \neq 0$, it follows that

$$
\gamma \int_{\Omega}\left|\nabla G_{k}\left(u_{n}\right)\right|^{2} d x \leqslant \int_{\Omega} f G_{k}\left(u_{n}\right) d x .
$$

Starting from inequality (31), Theorem 4.2 in [13] shows that there exists a constant $C$ (independent of $n$ ), such that

$$
\left\|u_{n}\right\|_{L^{\infty}(\Omega)} \leqslant C\|f\|_{L^{m}(\Omega)},
$$

which implies that $u$ belongs to $L^{\infty}(\Omega)$.

To prove (ii), noting that if $m=2 N /\left(N+2+(N-2) \alpha^{-}\right)$, $s=2 N /(N-2)=2^{*}$, since $u \in H_{0}^{1}(\Omega)$, the result when $m=2 N /\left(N+2+(N-2) \alpha^{-}\right)$is true by Sobolev Embedding Theorem. If $2 N /\left(N+2+(N-2) \alpha^{-}\right)<m<N / 2$, letting $\delta>1$ and choosing $u_{n}^{2 \delta-1}$ as a test function in (7), using Hölder's inequality, we get that

$$
\begin{aligned}
& \gamma(2 \delta-1) \int_{\Omega}\left|\nabla u_{n}\right|^{2} u_{n}^{2 \delta-2} d x \leqslant \int_{\Omega} \frac{f u_{n}^{2 \delta-1}}{\left(u_{n}+1 / n\right)^{\alpha(x)}} d x \\
& \leqslant \int_{\left\{x \in \Omega, u_{n} \geqslant 1\right\}} \frac{f u_{n}^{2 \delta-1}}{u_{n}^{\alpha^{-}}} d x+\int_{\left\{x \in \Omega, u_{n}<1\right\}} \frac{f u_{n}^{2 \delta-1}}{u_{n}^{\alpha^{+}}} d x \\
& \leqslant \int_{\Omega} f u_{n}^{2 \delta-1-\alpha^{-}} d x+\int_{\Omega} f u_{n}^{2 \delta-1-\alpha^{+}} d x \leqslant\|f\|_{L^{m}(\Omega)} \\
& \cdot\left(\int_{\Omega} u_{n}^{\left(2 \delta-1-\alpha^{-}\right) m^{\prime}} d x\right)^{1 / m^{\prime}}+\|f\|_{L^{m}(\Omega)} \\
& \cdot\left(\int_{\Omega} u_{n}^{\left(2 \delta-1-\alpha^{+}\right) m^{\prime}} d x\right)^{1 / m^{\prime}}=\|f\|_{L^{m}(\Omega)} \\
& \cdot\left(\left(\int_{\Omega} u_{n}^{\left(2 \delta-1-\alpha^{-}\right) m^{\prime}} d x\right)^{1 / m^{\prime}}\right. \\
& +|\Omega|^{\left(\alpha^{+}-\alpha^{-}\right) /\left(2 \delta-1-\alpha^{-}\right) m^{\prime}} \\
& \left..\left(\int_{\Omega} u_{n}^{\left(2 \delta-1-\alpha^{-}\right) m^{\prime}} d x\right)^{\left(2 \delta-1-\alpha^{+}\right) /\left(2 \delta-1-\alpha^{-}\right) m^{\prime}}\right) .
\end{aligned}
$$

By Sobolev inequality (on the left hand side), we have that

$$
\begin{aligned}
\int_{\Omega}\left|\nabla u_{n}\right|^{2} u_{n}^{2 \delta-2} d x & =\frac{1}{\delta^{2}} \int_{\Omega}\left|\nabla u_{n}^{\delta}\right|^{2} d x \\
& \geqslant \frac{S}{\delta^{2}}\left(\int_{\Omega} u_{n}^{2^{*} \delta} d x\right)^{2 / 2^{*}},
\end{aligned}
$$


where $S$ is the constant of the Sobolev embedding; combining with (33) and (34), we have that

$$
\begin{aligned}
& \frac{S \gamma(2 \delta-1)}{\delta^{2}}\left(\int_{\Omega} u_{n}^{2^{*} \delta} d x\right)^{2 / 2^{*}} \leqslant\|f\|_{L^{m}(\Omega)} \\
& \cdot\left(\left(\int_{\Omega} u_{n}^{\left(2 \delta-1-\alpha^{-}\right) m^{\prime}} d x\right)^{1 / m^{\prime}}\right. \\
& +|\Omega|^{\left(\alpha^{+}-\alpha^{-}\right) /\left(2 \delta-1-\alpha^{-}\right) m^{\prime}} \\
& \left.\cdot\left(\int_{\Omega} u_{n}^{\left(2 \delta-1-\alpha^{-}\right) m^{\prime}} d x\right)^{\left(2 \delta-1-\alpha^{+}\right) /\left(2 \delta-1-\alpha^{-}\right) m^{\prime}}\right) .
\end{aligned}
$$

We choose $\delta$ in such a way that $2^{*} \delta=\left(2 \delta-1-\alpha^{-}\right) m^{\prime}$; that is,

$$
\delta=\frac{\left(1+\alpha^{-}\right) m(N-2)}{2(N-2 m)}
$$

which yields that $\delta>1$ if and only if $2 N /(N+2+(N-$ 2) $\left.\alpha^{-}\right)<m<N / 2$, and that $2^{*} \delta=N m\left(1+\alpha^{-}\right) /(N-2 m)=s$. Therefore, (35) becomes

$$
\begin{aligned}
& \left(\int_{\Omega} u_{n}^{s} d x\right)^{2 / 2^{*}} \leqslant \frac{\delta^{2}}{S \gamma(2 \delta-1)}\|f\|_{L^{m}(\Omega)} \\
& \cdot\left(\left(\int_{\Omega} u_{n}^{s} d x\right)^{1 / m^{\prime}}+|\Omega|^{\left(\alpha^{+}-\alpha^{-}\right) /\left(2 \delta-1-\alpha^{-}\right) m^{\prime}}\right. \\
& \left.\cdot\left(\int_{\Omega} u_{n}^{s} d x\right)^{\left(2 \delta-1-\alpha^{+}\right) /\left(2 \delta-1-\alpha^{-}\right) m^{\prime}}\right)
\end{aligned}
$$

which implies that

$$
\begin{gathered}
\left(\int_{\Omega} u_{n}^{s} d x\right)^{\left(1+\alpha^{+}\right) / 2^{*} \delta} \leqslant \frac{\delta^{2}}{S \gamma(2 \delta-1)}\|f\|_{L^{m}(\Omega)} \\
\cdot\left(\left(\int_{\Omega} u_{n}^{s} d x\right)^{\left(\alpha^{+}-\alpha^{-}\right) / 2^{*} \delta}+|\Omega|^{\left(\alpha^{+}-\alpha^{-}\right) / 2^{*} \delta}\right) .
\end{gathered}
$$

Using Young's inequality on the right hand side in (38), we have that

$$
\begin{aligned}
& \left(\int_{\Omega} u_{n}^{s} d x\right)^{\left(1+\alpha^{+}\right) / 2^{*} \delta} \leqslant \frac{\delta^{2}}{S \gamma(2 \delta-1)}\|f\|_{L^{m}(\Omega)} \\
& \cdot\left(\varepsilon\left(\int_{\Omega} u_{n}^{s} d x\right)^{\left(1+\alpha^{+}\right) / 2^{*} \delta}+\varepsilon^{-\left(\alpha^{+}-\alpha^{-}\right) /\left(1+\alpha^{-}\right)}\right. \\
& \left.\quad+|\Omega|^{\left(\alpha^{+}-\alpha^{-}\right) / 2^{*} \delta}\right),
\end{aligned}
$$

where $\varepsilon=S \gamma(2 \delta-1) / 2 \delta^{2}\|f\|_{L^{m}(\Omega)}$. Thus, we get that

$$
\begin{aligned}
& \left(\int_{\Omega} u_{n}^{s} d x\right)^{\left(1+\alpha^{+}\right) / 2^{*} \delta} \\
& \quad \leqslant \frac{2 \delta^{2}\|f\|_{L^{m}(\Omega)}}{S \gamma(2 \delta-1)}\left(\left(\frac{2 \delta^{2}\|f\|_{L^{m}(\Omega)}}{S \gamma(2 \delta-1)}\right)^{\left(\alpha^{+}-\alpha^{-}\right) /\left(1+\alpha^{-}\right)}\right. \\
& \left.\quad+|\Omega|^{\left(\alpha^{+}-\alpha^{-}\right) / 2^{*} \delta}\right)
\end{aligned}
$$

Therefore, we know that $u_{n}$ is bounded in $L^{s}(\Omega)$, and so does $u \in L^{s}(\Omega)$.

Theorem 9. Suppose that $f \in L^{m}(\Omega),\left(1+\alpha^{+}\right) N /\left(\left(1+\alpha^{-}\right)(N-\right.$ $\left.2)+2\left(1+\alpha^{+}\right)\right) \leqslant m<2 N /\left(N+2+(N-2) \alpha^{-}\right)$, and $0<\alpha^{-} \leqslant$ $\alpha(x) \leqslant \alpha^{+}<1$. Then problem (1) has a solution $u$ in $W_{0}^{1, q}(\Omega)$, $q=N m\left(1+\alpha^{-}\right) /\left(N-m\left(1-\alpha^{-}\right)\right)$.

Proof. The lines of our proof are that if we can prove that $u_{n}$ is bounded in $W_{0}^{1, q}(\Omega)$ (with $q$ as in the statement), the existence of a solution $u$ in $W_{0}^{1, q}(\Omega)$ of (1) will be proved by passing to the limit in (7) as in the proof of Theorem 7. To prove that $u_{n}$ is bounded in $W_{0}^{1, q}(\Omega)$, we begin by proving that it is bounded in $L^{s}(\Omega)$, with $s=N m\left(1+\alpha^{-}\right) /(N-2 m)$. To attain this goal, we choose $u_{n}^{2 \delta-1}$ as a test function in (7) as in the statement of Lemma 8 , where $\left(1+\alpha^{+}\right) / 2 \leqslant \delta<1$; however $\nabla u_{n}^{2 \delta-1}$ will be singular at $u_{n}=0$, and therefore, we choose $\left(u_{n}+\varepsilon\right)^{2 \delta-1}-\varepsilon^{2 \delta-1}$ as a test function in (7), where $\varepsilon<1 / n$ for $n$ fixed; by (2) and $f_{n} \leqslant f$, we have that

$$
\begin{gathered}
\gamma(2 \delta-1) \int_{\Omega}\left|\nabla u_{n}\right|^{2}\left(u_{n}+\varepsilon\right)^{2 \delta-2} d x \\
\leqslant \int_{\Omega} f\left(u_{n}+\varepsilon\right)^{2 \delta-1-\alpha^{-}} d x \\
+\int_{\Omega} f\left(u_{n}+\varepsilon\right)^{2 \delta-1-\alpha^{+}} d x .
\end{gathered}
$$

By Sobolev Embedding Theorem $\left(H_{0}^{1}(\Omega) \hookrightarrow L^{2^{*}}(\Omega)\right)$ on the left hand side, it follows that

$$
\begin{aligned}
& \int_{\Omega}\left|\nabla u_{n}\right|^{2}\left(u_{n}+\varepsilon\right)^{2 \delta-2} d x \\
& \quad=\int_{\Omega} \frac{\left|\nabla\left(\left(u_{n}+\varepsilon\right)^{\delta}-\varepsilon^{\delta}\right)\right|^{2}}{\delta^{2}} d x \\
& \geqslant \frac{S}{\delta^{2}}\left(\int_{\Omega}\left(\left(u_{n}+\varepsilon\right)^{\delta}-\varepsilon^{\delta}\right)^{2^{*}} d x\right)^{2 / 2^{*}}
\end{aligned}
$$


where $S$ is the best constant of the Sobolev Embedding Theorem. Combining (41) with (42), we have that

$$
\begin{aligned}
& \frac{S \gamma(2 \delta-1)}{\delta^{2}}\left(\int_{\Omega}\left(\left(u_{n}+\varepsilon\right)^{\delta}-\varepsilon^{\delta}\right)^{2^{*}} d x\right)^{2 / 2^{*}} \\
& \leqslant \int_{\Omega} f\left(u_{n}+\varepsilon\right)^{2 \delta-1-\alpha^{-}} d x \\
& \quad+\int_{\Omega} f\left(u_{n}+\varepsilon\right)^{2 \delta-1-\alpha^{+}} d x .
\end{aligned}
$$

Using Hölder's inequality on the right hand side, we get

$$
\begin{aligned}
& \frac{S \gamma(2 \delta-1)}{\delta^{2}}\left(\int_{\Omega}\left(\left(u_{n}+\varepsilon\right)^{\delta}-\varepsilon^{\delta}\right)^{2^{*}} d x\right)^{2 / 2^{*}} \\
& \leqslant\|f\|_{L^{m}(\Omega)}\left(\int_{\Omega}\left(u_{n}+\varepsilon\right)^{\left(2 \delta-1-\alpha^{-}\right) m^{\prime}} d x\right)^{1 / m^{\prime}} \\
& \quad+|\Omega|^{\left(\alpha^{+}-\alpha^{-}\right) /\left(2 \delta-1-\alpha^{-}\right) m^{\prime}}\|f\|_{L^{m}(\Omega)} \\
& \cdot\left(\int_{\Omega}\left(u_{n}+\varepsilon\right)^{\left(2 \delta-1-\alpha^{-}\right) m^{\prime}} d x\right)^{\left(2 \delta-1-\alpha^{+}\right) /\left(2 \delta-1-\alpha^{-}\right) m^{\prime}} .
\end{aligned}
$$

Letting $\varepsilon \rightarrow 0$, we get (35); that is,

$$
\begin{aligned}
& \left(\int_{\Omega} u_{n}^{2^{*} \delta} d x\right)^{2 / 2^{*}} \leqslant \frac{\delta^{2}}{S \gamma(2 \delta-1)}\|f\|_{L^{m}(\Omega)} \\
& \cdot\left(\left(\int_{\Omega} u_{n}^{\left(2 \delta-1-\alpha^{-}\right) m^{\prime}} d x\right)^{1 / m^{\prime}}\right. \\
& +|\Omega|^{\left(\alpha^{+}-\alpha^{-}\right) /\left(2 \delta-1-\alpha^{-}\right) m^{\prime}} \\
& \left.\cdot\left(\int_{\Omega} u_{n}^{\left(2 \delta-1-\alpha^{-}\right) m^{\prime}} d x\right)^{\left(2 \delta-1-\alpha^{+}\right) /\left(2 \delta-1-\alpha^{-}\right) m^{\prime}}\right)
\end{aligned}
$$

where $\delta$ is chosen in such a way that $2^{*} \delta=\left(2 \delta-1-\alpha^{-}\right) m^{\prime}$; that is,

$$
\delta=\frac{\left(1+\alpha^{-}\right)(N-2) m}{2(N-2 m)} .
$$

If $m=\left(1+\alpha^{+}\right) N /\left(\left(1+\alpha^{-}\right)(N-2)+2\left(1+\alpha^{+}\right)\right)$, choosing $\delta=\left(1+\alpha^{+}\right) / 2$ in (43), and letting $\varepsilon \rightarrow 0$, we have that

$$
\begin{aligned}
& \left(\int_{\Omega} u_{n}^{2^{*} \delta} d x\right)^{2 / 2^{*}} \\
& \quad \leqslant \frac{\delta^{2}}{S \gamma(2 \delta-1)}\left(\int_{\Omega} f u_{n}^{2 \delta-1-\alpha^{-}} d x+\int_{\Omega} f d x\right) .
\end{aligned}
$$

Using Hölder's inequality and Young's inequality, we get

$$
\begin{aligned}
& \left(\int_{\Omega} u_{n}^{2^{*} \delta} d x\right)^{2 / 2^{*}} \leqslant \frac{\delta^{2}}{S \gamma(2 \delta-1)}\left(\|f\|_{L^{m}(\Omega)}\right. \\
& \left.\cdot\left(\int_{\Omega} u_{n}^{\left(2 \delta-1-\alpha^{-}\right) m^{\prime}} d x\right)^{1 / m^{\prime}}+\int_{\Omega} f d x\right) \\
& \leqslant \frac{\delta^{2}}{S \gamma(2 \delta-1)}\left(\|f\|_{L^{m}(\Omega)}\right. \\
& \left.\cdot\left(\varepsilon\left(\int_{\Omega} u_{n}^{2^{*} \delta} d x\right)^{2 / 2^{*}}+\varepsilon^{-2^{*} /\left(2 m^{\prime}-2^{*}\right)}\right)+\int_{\Omega} f d x\right),
\end{aligned}
$$

where $\varepsilon=S \gamma(2 \delta-1) / 2 \delta^{2}\|f\|_{L^{m}(\Omega)}$. Thus we have that

$$
\begin{gathered}
\left(\int_{\Omega} u_{n}^{2^{*} \delta} d x\right)^{2 / 2^{*}} \leqslant \frac{2 \delta^{2}}{S \gamma(2 \delta-1)}\left(\|f\|_{L^{m}(\Omega)}\right. \\
\left.\cdot\left(\frac{2 \delta^{2}\|f\|_{L^{m}(\Omega)}}{S \gamma(2 \delta-1)}\right)^{2^{*} /\left(2 m^{\prime}-2^{*}\right)}+\int_{\Omega} f d x\right) .
\end{gathered}
$$

Therefore we obtain that $u_{n}$ is bounded in $L^{N\left(1+\alpha^{+}\right) /(N-2)}(\Omega)$, where $N\left(1+\alpha^{+}\right) /(N-2)$ is the value of $s$ for $m=(1+$ $\left.\alpha^{+}\right) N /\left(\left(1+\alpha^{-}\right)(N-2)+2\left(1+\alpha^{+}\right)\right)$.

If $\left(1+\alpha^{+}\right) N /\left(\left(1+\alpha^{-}\right)(N-2)+2\left(1+\alpha^{+}\right)\right)<m<2 N /(N+$ $\left.2+(N-2) \alpha^{-}\right)$, it is clear that the inequality on $m$ holds true if and only if $\left(1+\alpha^{+}\right) / 2<\delta<1$, starting from (35) and arguing as in the proof of Lemma 8 , we also get that $u_{n}$ is bounded in $L^{s}(\Omega)$ with $s=N m\left(1+\alpha^{-}\right) /(N-2 m)$.

The right hand side of (41) is bounded with respect to $n$ (and $\varepsilon$, which we take smaller than 1 ) by using the estimate on $u_{n}$ in $L^{\mathcal{S}}(\Omega)$ and the choice of $\delta$.

Since $\delta<1$,

$$
\int_{\Omega} \frac{\left|\nabla u_{n}\right|^{2}}{\left(u_{n}+\varepsilon\right)^{2-2 \delta}} d x=\int_{\Omega}\left|\nabla u_{n}\right|^{2}\left(u_{n}+\varepsilon\right)^{2 \delta-2} d x \leqslant C .
$$

If $q=N m\left(1+\alpha^{-}\right) /\left(N-m\left(1-\alpha^{-}\right)\right)<2$, by Hölder's inequality, we have that

$$
\begin{gathered}
\int_{\Omega}\left|\nabla u_{n}\right|^{q} d x=\int_{\Omega} \frac{\left|\nabla u_{n}\right|^{q}}{\left(u_{n}+\varepsilon\right)^{(1-\delta) q}}\left(u_{n}+\varepsilon\right)^{(1-\delta) q} d x \\
\leqslant\left(\int_{\Omega}\left(\frac{\left|\nabla u_{n}\right|^{q}}{\left(u_{n}+\varepsilon\right)^{(1-\delta) q}}\right)^{2 / q} d x\right)^{q / 2} \\
\cdot\left(\int_{\Omega}\left(u_{n}+\varepsilon\right)^{2(1-\delta) q /(2-q)} d x\right)^{1-q / 2}
\end{gathered}
$$




$$
\begin{aligned}
& =\left(\int_{\Omega} \frac{\left|\nabla u_{n}\right|^{2}}{\left(u_{n}+\varepsilon\right)^{2(1-\delta)}} d x\right)^{q / 2} \\
& \cdot\left(\int_{\Omega}\left(u_{n}+\varepsilon\right)^{2(1-\delta) q /(2-q)} d x\right)^{1-q / 2} \\
& \leqslant C\left(\int_{\Omega}\left(u_{n}+\varepsilon\right)^{2(1-\delta) q /(2-q)} d x\right)^{1-q / 2} .
\end{aligned}
$$

The choice of $\delta$ and the value of $q$ are such that 2(1 $\delta) q /(2-q)=s$, so that the right hand side of (51) is bounded with respect to $n$ and $\varepsilon$. Hence, $u_{n}$ is bounded in $W_{0}^{1, q}(\Omega)$.

Theorem 10. Suppose that $f \in L^{m}(\Omega), 1 /\left(2 \delta-\alpha^{+}\right)<m<$ $\left(\left(1+\alpha^{+}\right) N /\left(\left(1+\alpha^{-}\right)(N-2)+2\left(1+\alpha^{+}\right)\right)\right)$with $\left(1+\alpha^{-}\right) / 2<$ $\delta<\left(1+\alpha^{+}\right) / 2$, and $0<\alpha^{-} \leqslant \alpha(x) \leqslant \alpha^{+}<1$. Then problem (1) has a solution $u$ in $W_{0}^{1, q}(\Omega), q=N m\left(1+\alpha^{-}\right) /\left(N-m\left(1-\alpha^{-}\right)\right)$.

Proof. The lines of our proof are similar to that in the proof of Theorem 9 . We also begin by proving that $u_{n}$ is bounded in $L^{s}(\Omega)$, with $s=N m\left(1+\alpha^{-}\right) /(N-2 m)$. To this aim, we also choose $\left(u_{n}+\varepsilon\right)^{2 \delta-1}-\varepsilon^{2 \delta-1}$ as a test function in (7), where $\left(1+\alpha^{-}\right) / 2<\delta<\left(1+\alpha^{+}\right) / 2, \varepsilon<1 / n$ for $n$ fixed. Since $f_{n} \leqslant f$ and (2), we have that

$$
\begin{gathered}
\gamma(2 \delta-1) \int_{\Omega}\left|\nabla u_{n}\right|^{2}\left(u_{n}+\varepsilon\right)^{2 \delta-2} d x \\
\leqslant \int_{\Omega} f\left(u_{n}+\varepsilon\right)^{2 \delta-1-\alpha^{-}} d x \\
+\int_{\Omega} f\left(u_{n}+\varepsilon\right)^{2 \delta-1-\alpha^{+}} d x .
\end{gathered}
$$

Using Sobolev Embedding Theorem $\left(H_{0}^{1}(\Omega) \hookrightarrow L^{2^{*}}(\Omega)\right)$ on the left hand side, it follows that

$$
\begin{aligned}
& \frac{S \gamma(2 \delta-1)}{\delta^{2}}\left(\int_{\Omega}\left(\left(u_{n}+\varepsilon\right)^{\delta}-\varepsilon^{\delta}\right)^{2^{*}} d x\right)^{2 / 2^{*}} \\
& \leqslant \int_{\Omega} f\left(u_{n}+\varepsilon\right)^{2 \delta-1-\alpha^{-}} d x \\
& \quad+\int_{\Omega} f\left(u_{n}+\varepsilon\right)^{2 \delta-1-\alpha^{+}} d x
\end{aligned}
$$

where $S$ is the constant of the Sobolev Embedding Theorem.

Using Hölder's inequality and Lemma 5 on the right hand side, we get

$$
\begin{aligned}
& \frac{S \gamma(2 \delta-1)}{\delta^{2}}\left(\int_{\Omega}\left(\left(u_{n}+\varepsilon\right)^{\delta}-\varepsilon^{\delta}\right)^{2^{*}} d x\right)^{2 / 2^{*}} \\
& \leqslant \int_{\Omega} f\left(u_{n}+\varepsilon\right)^{2 \delta-1-\alpha^{-}} d x \\
& \quad+\int_{\Omega} \frac{f}{\left(u_{n}+\varepsilon\right)^{-2 \delta+\alpha^{+}+1}} d x
\end{aligned}
$$

$$
\begin{aligned}
& \leqslant \int_{\Omega} f\left(u_{n}+\varepsilon\right)^{2 \delta-1-\alpha^{-}} d x+\int_{\Omega} \frac{f}{u_{1}^{-2 \delta+\alpha^{+}+1}} d x \\
& \leqslant\|f\|_{L^{m}(\Omega)}\left(\int_{\Omega}\left(u_{n}+\varepsilon\right)^{\left(2 \delta-1-\alpha^{-}\right) m^{\prime}} d x\right)^{1 / m^{\prime}} \\
& +\|f\|_{L^{m}(\Omega)}\left\|u_{1}^{2 \delta-1-\alpha^{+}}\right\|_{L^{m^{\prime}}(\Omega)} \\
& \leqslant\|f\|_{L^{m}(\Omega)}\left(\int_{\Omega}\left(u_{n}+\varepsilon\right)^{\left(2 \delta-1-\alpha^{-}\right) m^{\prime}} d x\right)^{1 / m^{\prime}} \\
& +C\|f\|_{L^{m}(\Omega)} .
\end{aligned}
$$

Letting $\varepsilon \rightarrow 0$, we obtain that

$$
\begin{gathered}
\left(\int_{\Omega} u_{n}^{2^{*} \delta} d x\right)^{2 / 2^{*}} \leqslant \frac{\delta^{2}}{S \gamma(2 \delta-1)}\|f\|_{L^{m}(\Omega)} \\
\cdot\left(\left(\int_{\Omega} u_{n}^{\left(2 \delta-1-\alpha^{-}\right) m^{\prime}} d x\right)^{1 / m^{\prime}}+C\right),
\end{gathered}
$$

where $\delta$ is chosen in such a way that $2^{*} \delta=\left(2 \delta-1-\alpha^{-}\right) m^{\prime}$; that is

$$
\delta=\frac{\left(1+\alpha^{-}\right)(N-2) m}{2(N-2 m)}
$$

If $1<m<\left(1+\alpha^{+}\right) N /\left(\left(1+\alpha^{-}\right)(N-2)+2\left(1+\alpha^{+}\right)\right)$, it is clear that the inequality on $m$ holds true if and only if $\left(1+\alpha^{-}\right) / 2<\delta<\left(1+\alpha^{+}\right) / 2$, and arguing as to the case $m=(1+$ $\left.\alpha^{+}\right) N /\left(\left(1+\alpha^{-}\right)(N-2)+2\left(1+\alpha^{+}\right)\right)$in the proof of Theorem 9 , we also obtain that $u_{n}$ is bounded in $L^{s}(\Omega)$, with $s=N m(1+$ $\left.\alpha^{-}\right) /(N-2 m)$.

Since $\delta<1$,

$$
\int_{\Omega} \frac{\left|\nabla u_{n}\right|^{2}}{\left(u_{n}+\varepsilon\right)^{2-2 \delta}} d x=\int_{\Omega}\left|\nabla u_{n}\right|^{2}\left(u_{n}+\varepsilon\right)^{2 \delta-2} d x \leqslant C .
$$

If $q=N m\left(1+\alpha^{-}\right) /\left(N-m\left(1-\alpha^{-}\right)\right)<2$, similarly to the proof of Theorem 9, we have by Hölder's inequality that

$$
\int_{\Omega}\left|\nabla u_{n}\right|^{q} d x \leqslant C\left(\int_{\Omega}\left(u_{n}+\varepsilon\right)^{2(1-\delta) q /(2-q)} d x\right)^{1-q / 2} .
$$

Since the choice of $\delta$ and the value of $q$, the right hand side of the above inequality is bounded with respect to $n$ and $\varepsilon$. Hence, $u_{n}$ is bounded in $W_{0}^{1, q}(\Omega)$.

\section{The Case $1<\alpha^{-} \leqslant \alpha(x) \leqslant \alpha^{+}$}

The case $1<\alpha^{-} \leqslant \alpha(x) \leqslant \alpha^{+}$has many analogies with the case $0<\alpha^{-}<\alpha^{+}<1$. In this case, we can also prove that $u_{n}$ is bounded in $H_{0}^{1}(\Omega)$ only if $f$ is more regular than $L^{1}(\Omega)$ and $\alpha^{+}$and $\alpha^{-}$are close to 1 .

Lemma 11. Suppose that $f \in L^{m}(\Omega)(m>1)$, and let $u_{n}$ be the solution of (7) with $1<\alpha^{-}<\alpha^{+}<2-1 / m$. Then $u_{n}$ is bounded in $H_{0}^{1}(\Omega)$. 
Proof. Taking $u_{n}$ as a test function in (7), by (2), we obtain that

$$
\gamma \int_{\Omega}\left|\nabla u_{n}\right|^{2} d x \leqslant \int_{\Omega} \frac{f}{u_{n}^{\alpha(x)-1}} d x .
$$

Using Lemmas 2 and 3, we know that $u_{n} \geqslant u_{1}$ and there exists a constant $M>0$ s.t. $u_{1} \leqslant M$. Hence $\left(M / u_{1}\right)^{\alpha(x)-1} \leqslant$ $\left(M / u_{1}\right)^{\alpha^{+}-1}$, and we have

$$
\begin{aligned}
\gamma \int_{\Omega}\left|\nabla u_{n}\right|^{2} d x & \leqslant \int_{\Omega} \frac{f}{u_{n}^{\alpha(x)-1}} d x \leqslant \int_{\Omega} \frac{f}{u_{1}^{\alpha(x)-1}} d x \\
& \leqslant\left(1+M^{\alpha^{+}-\alpha^{-}}\right) \int_{\Omega} \frac{f}{u_{1}^{\alpha^{+}-1}} d x .
\end{aligned}
$$

Using Hölder's inequality on the right hand side, and Lemma 5, we obtain

$$
\begin{aligned}
& \gamma \int_{\Omega}\left|\nabla u_{n}\right|^{2} d x \leqslant\left(1+M^{\alpha^{+}-\alpha^{-}}\right) \int_{\Omega} \frac{f}{u_{1}^{\alpha^{+}-1}} d x \\
& \leqslant\left(1+M^{\alpha^{+}-\alpha^{-}}\right)\|f\|_{L^{m}(\Omega)}\left(\int_{\Omega} \frac{1}{u_{1}^{\left(\alpha^{+}-1\right) m^{\prime}}} d x\right)^{1 / m^{\prime}} \\
& \quad \leqslant C\left(1+M^{\alpha^{+}-\alpha^{-}}\right)\|f\|_{L^{m}(\Omega)} .
\end{aligned}
$$

Therefore, $u_{n}$ is bounded in $H_{0}^{1}(\Omega)$.

Once we have the boundedness of $u_{n}$, we can prove the following existence theorem along the lines of Theorem 7.

Theorem 12. Suppose that $f \in L^{m}(\Omega)(m>1)$ and $1<\alpha^{-}<$ $\alpha^{+}<2-1 / m$. Then problem (1) has a solution $u$ in $H_{0}^{1}(\Omega)$.

The summability of $u$ can be proved along the lines of Lemma 8 with little changes.

Lemma 13. Suppose that $f \in L^{m}(\Omega)(m>1)$ and $1<\alpha^{-}<$ $\alpha^{+}<2-1 / m$. Then the solution $u$ of (1) given by Theorem 12 is such that

(i) if $m>N / 2$, then $u \in L^{\infty}(\Omega)$;

(ii) if $N\left(1+\alpha^{+}\right) /\left(\left(1+\alpha^{-}\right)(N-2)+2\left(1+\alpha^{+}\right)\right) \leqslant m<N / 2$, then $u \in L^{s}(\Omega), s=N m\left(1+\alpha^{-}\right) /(N-2 m)$.

Proof. The proof of (i) is similar to the proof of Lemma 8(i); we omit the details here.

To prove (ii) we choose $u_{n}^{2 \delta-1}$ as a test function with $\delta \geqslant$ $\left(1+\alpha^{+}\right) / 2$ in (7); similarly to the proof of Lemma 8 , we obtain that

$$
\begin{aligned}
& \frac{S \gamma(2 \delta-1)}{\delta^{2}}\left(\int_{\Omega} u_{n}^{2^{*} \delta} d x\right)^{2 / 2^{*}} \\
& \leqslant \int_{\Omega} f u_{n}^{2 \delta-1-\alpha^{-}} d x+\int_{\Omega} f u_{n}^{2 \delta-1-\alpha^{+}} d x
\end{aligned}
$$

If $m=N\left(1+\alpha^{+}\right) /\left(\left(1+\alpha^{-}\right)(N-2)+2\left(1+\alpha^{+}\right)\right)$, choosing $\delta=\left(1+\alpha^{+}\right) / 2$ in (62), by Hölder's inequality, we get

$$
\begin{aligned}
& \frac{S \gamma(2 \delta-1)}{\delta^{2}}\left(\int_{\Omega} u_{n}^{2^{*} \delta} d x\right)^{2 / 2^{*}} \\
& \leqslant\|f\|_{L^{m}(\Omega)}\left(\int_{\Omega} u_{n}^{\left(2 \delta-1-\alpha^{-}\right) m^{\prime}} d x\right)^{1 / m^{\prime}} \\
& \quad+|\Omega|^{1-1 / m}\|f\|_{L^{m}(\Omega)} .
\end{aligned}
$$

We choose $\delta$ in such a way that $2^{*} \delta=\left(2 \delta-1-\alpha^{-}\right) m^{\prime}$; that is, $\delta=\left(1+\alpha^{-}\right) m(N-2) / 2(N-2 m)$. Since $m=N(1+$ $\left.\alpha^{+}\right) /\left(\left(1+\alpha^{-}\right)(N-2)+2\left(1+\alpha^{+}\right)\right)$being $2 / 2^{*}>1 / m^{\prime}$, it follows that $u_{n}$ is bounded in $L^{N\left(1+\alpha^{+}\right) /(N-2)}(\Omega)$.

If $N\left(1+\alpha^{+}\right) /\left(\left(1+\alpha^{-}\right)(N-2)+2\left(1+\alpha^{+}\right)\right)<m<N / 2$, starting from inequality (62) and Hölder's inequality, we have that

$$
\begin{aligned}
& \frac{S \gamma(2 \delta-1)}{\delta^{2}}\left(\int_{\Omega} u_{n}^{2^{*} \delta} d x\right)^{2 / 2^{*}} \leqslant\|f\|_{L^{m}(\Omega)} \\
& \cdot\left(\left(\int_{\Omega} u_{n}^{\left(2 \delta-1-\alpha^{-}\right) m^{\prime}} d x\right)^{1 / m^{\prime}}\right. \\
& +|\Omega|^{\left(\alpha^{+}-\alpha^{-}\right) /\left(2 \delta-1-\alpha^{-}\right) m^{\prime}} \\
& \left.\cdot\left(\int_{\Omega} u_{n}^{\left(2 \delta-1-\alpha^{-}\right) m^{\prime}} d x\right)^{\left(2 \delta-1-\alpha^{+}\right) /\left(2 \delta-1-\alpha^{-}\right) m^{\prime}}\right) .
\end{aligned}
$$

We also choose $\delta$ in such a way that $2^{*} \delta=\left(2 \delta-1-\alpha^{-}\right) m^{\prime}$, which yields that $\delta>\left(1+\alpha^{+}\right) / 2$, if and only if $m>N(1+$ $\left.\alpha^{+}\right) /\left(\left(1+\alpha^{-}\right)(N-2)+2\left(1+\alpha^{+}\right)\right)$, and that $2^{*} \delta=s$. So, since $2 / 2^{*}>1 / m^{\prime}$ being $m<N / 2$, we have the boundedness of $u_{n}$ in $L^{s}(\Omega)$, and so does $u \in L^{s}(\Omega)$.

Moreover, we can only prove that a positive power of $u_{n}$ is bounded in $H_{0}^{1}(\Omega)$ only if $f$ is more regular than $L^{1}(\Omega)$ and $\alpha^{+}$is close to $\alpha^{-}$and we only have the boundedness of $u_{n}$ in $H_{\mathrm{loc}}^{1}(\Omega)$.

Lemma 14. Suppose that $f \in L^{m}(\Omega)(m>1)$, and let $u_{n}$ be the solution of (7) with $1<\alpha^{-} \leqslant \alpha(x) \leqslant \alpha^{+}$and $\alpha^{+}-\alpha^{-}<$ $1-1 / m$. Then $u_{n}^{\left(1+\alpha^{-}\right) / 2}$ is bounded in $H_{0}^{1}(\Omega)$, and $u_{n}$ is bounded in $H_{\mathrm{loc}}^{1}(\Omega)$ and in $L^{s}(\Omega)$, with $s=N\left(1+\alpha^{-}\right) /(N-2)$.

Proof. Taking $u_{n}^{\alpha^{-}}$as a test function in (7), since $u_{n}^{\alpha^{-}} /\left(u_{n}+\right.$ $1 / n)^{\alpha^{-}} \leqslant 1$ and $f_{n} \leqslant f$, by Lemma 5 and (2), we get that

$$
\begin{aligned}
& \alpha^{-} \gamma \int_{\Omega}\left|\nabla u_{n}\right|^{2} u_{n}^{\alpha^{-}-1} d x \\
& \quad \leqslant \int_{\Omega} \frac{f u_{n}^{\alpha^{-}}}{\left(u_{n}+1 / n\right)^{\alpha^{-}}} d x+\int_{\Omega} \frac{f u_{n}^{\alpha^{-}}}{u_{n}^{\alpha^{+}}} d x \\
& \quad \leqslant|\Omega|^{1-1 / m}\|f\|_{L^{m}(\Omega)}+\|f\|_{L^{m}(\Omega)} \int_{\Omega} \frac{1}{u_{n}^{\left(\alpha^{+}-\alpha^{-}\right) m^{\prime}}} d x \\
& \quad \leqslant|\Omega|^{1-1 / m}\|f\|_{L^{m}(\Omega)}+C\|f\|_{L^{m}(\Omega)} .
\end{aligned}
$$


Since

$$
\int_{\Omega}\left|\nabla u_{n}\right|^{2} u_{n}^{\alpha^{-}-1} d x=\frac{4}{\left(1+\alpha^{-}\right)^{2}} \int_{\Omega}\left|\nabla u_{n}^{\left(1+\alpha^{-}\right) / 2}\right|^{2} d x
$$

we have that

$$
\begin{aligned}
& \frac{4 \alpha^{-} \gamma}{\left(1+\alpha^{-}\right)^{2}} \int_{\Omega}\left|\nabla u_{n}^{\left(1+\alpha^{-}\right) / 2}\right|^{2} d x \\
& \quad \leqslant\left(C+|\Omega|^{1-1 / m}\right)\|f\|_{L^{m}(\Omega)} .
\end{aligned}
$$

Thus, we have that $u_{n}^{\left(1+\alpha^{-}\right) / 2}$ is bounded in $H_{0}^{1}(\Omega)$.

$$
\text { Applying Sobolev Embedding Theorem to }
$$
$u_{n}^{\left(1+\alpha^{-}\right) / 2}\left(H_{0}^{1}(\Omega) \hookrightarrow L^{2^{*}}(\Omega)\right)$, we have that

$$
S\left(\int_{\Omega}\left|u_{n}^{\left(1+\alpha^{-}\right) / 2}\right|^{2^{*}} d x\right)^{2 / 2^{*}} \leqslant \int_{\Omega}\left|\nabla u_{n}^{\left(1+\alpha^{-}\right) / 2}\right|^{2} d x
$$

where $S$ is the best constant of the Sobolev embedding. Since the boundedness of $u_{n}^{\left(1+\alpha^{-}\right) / 2}$ in $H_{0}^{1}(\Omega)$, we thus have the boundedness of $u_{n}$ in $L^{\mathcal{s}}(\Omega)$.

To prove the boundedness of $u_{n}$ in $H_{\text {loc }}^{1}(\Omega)$, we choose $u_{n} \varphi^{2}$ as a test function in (7), where $\varphi \in C_{0}^{1}(\Omega), \Omega^{\prime}=\{x \in$ $\Omega, \varphi \neq 0\}$. By (2) and (12), we have that

$$
\begin{aligned}
& \gamma \int_{\Omega}\left|\nabla u_{n}\right|^{2} \varphi^{2} d x+2 \int_{\Omega}\left(M(x) \nabla u_{n}\right) \cdot \nabla \varphi u_{n} \varphi d x \\
& \leqslant \int_{\Omega}\left(M(x) \nabla u_{n}\right) \nabla\left(u_{n} \varphi^{2}\right) d x \\
& \quad=\int_{\Omega} \frac{f_{n} u_{n} \varphi^{2}}{\left(u_{n}+1 / n\right)^{\alpha(x)}} d x \leqslant \int_{\Omega} \frac{f_{n} \varphi^{2}}{u_{n}^{\alpha(x)-1}} d x \\
& \quad \leqslant \int_{\Omega} \frac{f_{n} \varphi^{2}}{C_{\Omega^{\prime}}^{\alpha(x)-1} d x} \\
& \quad \leqslant \frac{1}{\min \left\{C_{\Omega^{\prime}}^{\alpha^{\prime}-1}, C_{\Omega^{\prime}}^{\alpha^{-}-1}\right\}} \int_{\Omega} f_{n} \varphi^{2} d x .
\end{aligned}
$$

By Young's inequality, we get that

$$
\begin{aligned}
& 2 \beta \int_{\Omega} \nabla u_{n} \cdot \nabla \varphi u_{n} \varphi d x \\
& \quad \leqslant \frac{\gamma}{2} \int_{\Omega}\left|\nabla u_{n}\right|^{2} \varphi^{2} d x+\frac{2 \beta^{2}}{\gamma} \int_{\Omega}|\nabla \varphi|^{2} u_{n}^{2} d x .
\end{aligned}
$$

Since $u_{n}$ is bounded in $L^{s}(\Omega)$ (where $s \geqslant 2$ ), by Hölder inequality, we obtain that

$$
\begin{aligned}
\frac{\gamma}{2} \int_{\Omega}\left|\nabla u_{n}\right|^{2} \varphi^{2} d x & \frac{1}{\min \left\{C_{\Omega^{\prime}}^{\alpha^{+}-1}, C_{\Omega^{\prime}}^{\alpha^{-}-1}\right\}} \int_{\Omega} f_{n} \varphi^{2} d x \\
& +\frac{2 \beta^{2}}{\gamma} \int_{\Omega}|\nabla \varphi|^{2} u_{n}^{2} d x \\
\leqslant & \frac{\|\varphi\|_{L^{\infty}(\Omega)}^{2}}{\min \left\{C_{\Omega^{\prime}}^{\alpha^{\prime}-1}, C_{\Omega^{\prime}}^{\alpha^{-}-1}\right\}} \int_{\Omega} f d x \\
& +\frac{2 \beta^{2}}{\gamma}\|\nabla \varphi\|_{L^{\infty}(\Omega)}^{2} \int_{\Omega} u_{n}^{2} d x \\
\leqslant & \frac{|\Omega|^{1-1 / m}\|\varphi\|_{L^{\infty}(\Omega)}^{2}}{\min \left\{C_{\Omega^{\prime}}^{\alpha^{+}-1}, C_{\Omega^{\prime}}^{\alpha^{-}-1}\right\}}\|\|_{L^{m}(\Omega)} \\
& +\frac{2 \beta^{2}}{\gamma}\|\nabla \varphi\|_{L^{\infty}(\Omega)}^{2}|\Omega|^{1-2 / s}\left(\int_{\Omega} u_{n}^{s} d x\right)^{2 / s} \\
\leqslant & C(f, \varphi),
\end{aligned}
$$

and hence $u_{n}$ is bounded in $H_{\text {loc }}^{1}(\Omega)$.

Once we have the boundedness of $u_{n}$, we can prove the following existence theorem along the lines of Theorem 7.

Theorem 15. Suppose that $f$ is a nonnegative function in $L^{m}(\Omega)(m>1),(f \neq \equiv 0), 1<\alpha^{-} \leqslant \alpha(x) \leqslant \alpha^{+}$and $\alpha^{+}-\alpha^{-}<1-1 / m$. Then problem (1) has a solution $u$ in $H_{\mathrm{loc}}^{1}(\Omega)$. Furthermore, $u^{\left(1+\alpha^{-}\right) / 2}$ belongs to $H_{0}^{1}(\Omega)$.

The summability of $u$ can be proved along the lines of Lemma 8 with little changes.

Lemma 16. Suppose that $f \in L^{m}(\Omega), 1<\alpha^{-} \leqslant \alpha(x) \leqslant \alpha^{+}$, and $\alpha^{+}-\alpha^{-}<1-1 / m$. Then the solution $u$ of (1) given by Theorem 15 is such that

(i) if $m>N / 2$, then $u \in L^{\infty}(\Omega)$;

(ii) if $N\left(1+\alpha^{+}\right) /\left(\left(1+\alpha^{-}\right)(N-2)+2\left(1+\alpha^{+}\right)\right) \leqslant m<N / 2$, then $u \in L^{s}(\Omega), s=N m\left(1+\alpha^{-}\right) /(N-2 m)$.

Proof. The proof of (i) is similar to the proof of Lemma 8(i); we omit the details here.

To prove (ii), we choose $u_{n}^{2 \delta-1}\left(\delta \geqslant\left(1+\alpha^{+}\right) / 2\right)$ as a test function in (7); applying (2) and Sobolev Embedding Theorem, we have that

$$
\begin{aligned}
& \frac{S \gamma(2 \delta-1)}{\delta^{2}}\left(\int_{\Omega} u_{n}^{2^{*} \delta} d x\right)^{2 / 2^{*}} \\
& \quad \leqslant \int_{\Omega} f u_{n}^{2 \delta-1-\alpha^{-}} d x+\int_{\Omega} f u_{n}^{2 \delta-1-\alpha^{+}} d x .
\end{aligned}
$$


If $m=N\left(1+\alpha^{+}\right) /\left(\left(1+\alpha^{-}\right)(N-2)+2\left(1+\alpha^{+}\right)\right)$, we choose $\delta=\left(1+\alpha^{+}\right) / 2$; using Hölder inequality, we have that

$$
\begin{aligned}
& \frac{S \gamma(2 \delta-1)}{\delta^{2}}\left(\int_{\Omega} u_{n}^{2^{*} \delta} d x\right)^{2 / 2^{*}} \\
& \leqslant\|f\|_{L^{m}(\Omega)}\left(\int_{\Omega} u_{n}^{\left(2 \delta-1-\alpha^{-}\right) m^{\prime}} d x\right)^{1 / m^{\prime}} \\
& \quad+|\Omega|^{1-1 / m}\|f\|_{L^{m}(\Omega)}
\end{aligned}
$$

We also choose $\delta$ in such a way that $2^{*} \delta=\left(2 \delta-1-\alpha^{-}\right) m^{\prime}$, since $m=N\left(1+\alpha^{+}\right) /\left(\left(1+\alpha^{-}\right)(N-2)+2\left(1+\alpha^{+}\right)\right)$being $2 / 2^{*}>$ $1 / m^{\prime}$, and we have the boundedness of $u_{n}$ in $L^{N\left(1+\alpha^{+}\right) /(N-2)}(\Omega)$ which is the value of $s$ for $m=N\left(1+\alpha^{+}\right) /\left(\left(1+\alpha^{-}\right)(N-2)+\right.$ $\left.2\left(1+\alpha^{+}\right)\right)$.

If $N\left(1+\alpha^{+}\right) /\left(\left(1+\alpha^{-}\right)(N-2)+2\left(1+\alpha^{+}\right)\right)<m<N / 2$, starting from (72) using Hölder inequality, we get that

$$
\begin{aligned}
& \frac{S \gamma(2 \delta-1)}{\delta^{2}}\left(\int_{\Omega} u_{n}^{2^{*} \delta} d x\right)^{2 / 2^{*}} \leqslant\|f\|_{L^{m}(\Omega)} \\
& \cdot\left(\int_{\Omega} u_{n}^{\left(2 \delta-1-\alpha^{-}\right) m^{\prime}} d x\right)^{1 / m^{\prime}}+\|f\|_{L^{m}(\Omega)} \\
& \cdot|\Omega|^{\left(\alpha^{+}-\alpha^{-}\right) /\left(2 \delta-1-\alpha^{-}\right) m^{\prime}} \\
& \cdot\left(\int_{\Omega} u_{n}^{\left(2 \delta-1-\alpha^{-}\right) m^{\prime}} d x\right)^{\left(2 \delta-1-\alpha^{+}\right) /\left(2 \delta-1-\alpha^{-}\right) m^{\prime}} .
\end{aligned}
$$

We also choose $\delta$ in such a way that $2^{*} \delta=\left(2 \delta-1-\alpha^{-}\right) m^{\prime}$, which yields that $\delta>\left(1+\alpha^{+}\right) / 2$, if and only if $m>N(1+$ $\left.\alpha^{+}\right) /\left(\left(1+\alpha^{-}\right)(N-2)+2\left(1+\alpha^{+}\right)\right)$, and that $2^{*} \delta=s$. Since $2 / 2^{*}>1 / m^{\prime}$ being $m<N / 2$, we have the boundedness of $u_{n}$ in $L^{s}(\Omega)$, and so does $u \in L^{s}(\Omega)$.

\section{The Case $0<\alpha^{-}<1<\alpha^{+}$}

If $\alpha^{-}<1<\alpha^{+}$, the boundedness of $u_{n}$ in $H_{0}^{1}(\Omega)$ can also be obtained only if $f$ is more regular than $L^{1}(\Omega)$, and the proof has many analogies with the case $0<\alpha^{-}<\alpha^{+}<1$. We have the following results.

Lemma 17. Suppose that $f \in L^{m}(\Omega)$, with $m=2 N /(N+2+$ $\left.(N-2) \alpha^{-}\right)$, and let $u_{n}$ be the solution of (7) with $0<\alpha^{-}<1<$ $\alpha^{+}<2-1 / m$. Then the sequence $\left\{u_{n}\right\}$ is bounded in $H_{0}^{1}(\Omega)$.

Proof. We choose $u_{n}$ as a test function in (7), by Hölder's inequality, (2), and Lemma 5 , since $f_{n} \leqslant f$, we have that

$$
\begin{aligned}
\gamma \int_{\Omega}\left|\nabla u_{n}\right|^{2} d x \leqslant & \|f\|_{L^{m}(\Omega)}\left(\int_{\Omega} u_{n}^{\left(1-\alpha^{-}\right) m^{\prime}} d x\right)^{1 / m^{\prime}} \\
& +\|f\|_{L^{m}(\Omega)}\left(\int_{\Omega} \frac{1}{u_{1}^{\left(\alpha^{+}-1\right) m^{\prime}}} d x\right)^{1 / m^{\prime}}
\end{aligned}
$$

$$
\begin{aligned}
\leqslant & \|f\|_{L^{m}(\Omega)}\left(\int_{\Omega} u_{n}^{\left(1-\alpha^{-}\right) m^{\prime}} d x\right)^{1 / m^{\prime}} \\
& +C\|f\|_{L^{m}(\Omega)} .
\end{aligned}
$$

Applying Sobolev Embedding Theorem on the left hand side, we get

$$
\begin{aligned}
S \gamma\left(\int_{\Omega} u_{n}^{2^{*}} d x\right)^{2 / 2^{*}} \leqslant & \|f\|_{L^{m}(\Omega)}\left(\int_{\Omega} u_{n}^{\left(1-\alpha^{-}\right) m^{\prime}} d x\right)^{1 / m^{\prime}} \\
& +C\|f\|_{L^{m}(\Omega)}
\end{aligned}
$$

Let $2^{*}=\left(1-\alpha^{-}\right) m^{\prime}$, and it follows that

$$
\begin{aligned}
S \gamma\left(\int_{\Omega} u_{n}^{2^{*}} d x\right)^{2 / 2^{*}} \leqslant & \|f\|_{L^{m}(\Omega)}\left(\int_{\Omega} u_{n}^{2^{*}} d x\right)^{1 / m^{\prime}} \\
& +C\|f\|_{L^{m}(\Omega)} .
\end{aligned}
$$

By Young's inequality, we get that

$$
\begin{aligned}
& S \gamma\left(\int_{\Omega} u_{n}^{2^{*}} d x\right)^{2 / 2^{*}} \\
& \quad \leqslant\|f\|_{L^{m}(\Omega)}\left(\varepsilon\left(\int_{\Omega} u_{n}^{2^{*}} d x\right)^{2 / 2^{*}}+\varepsilon^{-2^{*} /\left(2 m^{\prime}-2^{*}\right)}\right) \\
& \quad+C\|f\|_{L^{m}(\Omega)} .
\end{aligned}
$$

Thus, we have that

$$
\begin{aligned}
& \left(\int_{\Omega} u_{n}^{2^{*}} d x\right)^{2 / 2^{*}} \\
& \quad \leqslant \frac{\|f\|_{L^{m}(\Omega)}}{S \gamma-\|f\|_{L^{m}(\Omega)} \varepsilon}\left(\varepsilon^{-2^{*} /\left(2 m^{\prime}-2^{*}\right)}+C\right) .
\end{aligned}
$$

We choose $\varepsilon=S \gamma / 2\|f\|_{L^{m}(\Omega)}$ to get

$$
\begin{aligned}
& \left(\int_{\Omega} u_{n}^{2^{*}} d x\right)^{2 / 2^{*}} \\
& \quad \leqslant \frac{2\|f\|_{L^{m}(\Omega)}}{S \gamma}\left(\left(\frac{2\|f\|_{L^{m}(\Omega)}}{S \gamma}\right)^{2^{*} /\left(2 m^{\prime}-2^{*}\right)}+C\right) .
\end{aligned}
$$

So the boundedness of $u_{n}$ in $L^{2^{*}}(\Omega)$ is obtained; using the estimate and (75) again, we have the estimate of $u_{n}$ in $H_{0}^{1}(\Omega)$.

Once the boundedness of $u_{n}$ in $H_{0}^{1}(\Omega)$ is obtained, we can prove the following existence theorem.

Theorem 18. Suppose that $f \in L^{m}(\Omega)$ with $m=2 N /(N+2+$ $\left.(N-2) \alpha^{-}\right)$, and $0<\alpha^{-}<1<\alpha^{+}<2-1 / m$. Then problem (1) has a solution $u$ in $H_{0}^{1}(\Omega)$.

Lemma 19. Suppose that $f \in L^{m}(\Omega)$ with $m \geqslant 2 N /(N+2+$ $\left.(N-2) \alpha^{-}\right)$, and $\alpha^{-}<1<\alpha^{+}<2-1 / m$. Then the solution $u$ of (1) given by Theorem 18 is such that 
(i) if $m>N / 2$, then $u \in L^{\infty}(\Omega)$;

(ii) if $2 N /\left(N+2+(N-2) \alpha^{-}\right) \leqslant m<N / 2$, then $u \in L^{s}(\Omega)$, $s=N m\left(1+\alpha^{-}\right) /(N-2 m)$.

Proof. The proof of (i) is similar to that for Lemma 8(i), and we omit the details here.

To prove (ii), if $N\left(1+\alpha^{+}\right) /\left(\left(1+\alpha^{-}\right)(N-2)+2\left(1+\alpha^{+}\right)\right) \leqslant$ $m<N / 2$, the proof is identical to that for Lemma 13, and we also omit it here.

If $m=2 N /\left(N+2+(N-2) \alpha^{-}\right)$, we can prove the results by Sobolev Embedding Theorem.

If $2 N /\left(N+2+(N-2) \alpha^{-}\right)<m<N\left(1+\alpha^{+}\right) /\left(\left(1+\alpha^{-}\right)(N-\right.$ $\left.2)+2\left(1+\alpha^{+}\right)\right)$, we choose $1<\delta<\left(1+\alpha^{+}\right) / 2$, and we use once again $u_{n}^{2 \delta-1}$ as a test function in (7). Using $\delta>1>\left(1+\alpha^{-}\right) / 2$, as well as Hölder's inequality, Sobolev Embedding Theorem, Lemma 5, and (2), we get

$$
\begin{aligned}
& \frac{S \gamma(2 \delta-1)}{\delta^{2}}\left(\int_{\Omega} u_{n}^{2^{*} \delta} d x\right)^{2 / 2^{*}} \\
& \leqslant\|f\|_{L^{m}(\Omega)}\left(\int_{\Omega} u_{n}^{\left(2 \delta-1-\alpha^{-}\right) m^{\prime}} d x\right)^{1 / m^{\prime}}+C\|f\|_{L^{m}(\Omega)}
\end{aligned}
$$

The choice of $\delta$ in such a way that $2^{*} \delta=\left(2 \delta-1-\alpha^{-}\right) m^{\prime}$ yields that $1<\delta<\left(1+\alpha^{+}\right) / 2$, if and only if $2 N /(N+2+(N-$ $\left.2) \alpha^{-}\right)<m<N\left(1+\alpha^{+}\right) /\left(\left(1+\alpha^{-}\right)(N-2)+2\left(1+\alpha^{+}\right)\right)$, and that $2^{*} \delta=s$. The choice of $m<N / 2$ implies that $2 / 2^{*}>1 / m^{\prime}$. Thus we have the boundedness of $u_{n}$ in $L^{s}(\Omega)$, and so does the limit $u$ in $L^{s}(\Omega)$.

\section{Competing Interests}

The authors declare that they have no competing interests.

\section{Acknowledgments}

The project is supported by NSFC (11271154), by Key Lab of Symbolic Computation and Knowledge Engineering of Ministry of Education, and by the 985 Program of Jilin University.

\section{References}

[1] G. Dal Maso, F. Murat, L. Orsina, and A. Prignet, "Renormalized solutions of elliptic equations with general measure data," Annali della Scuola Normale Superiore di Pisa. Classe di Scienze. Serie IV, vol. 28, no. 4, pp. 741-808, 1999.

[2] A. Ambrosetti, H. Brezis, and G. Cerami, "Combined effects of concave and convex nonlinearities in some elliptic problems," Journal of Functional Analysis, vol. 122, no. 2, pp. 519-543, 1994.

[3] A. Nachman and A. Callegari, "A nonlinear singular boundary value problem in the theory of pseudoplastic fluids," SIAM Journal on Applied Mathematics, vol. 38, no. 2, pp. 275-281, 1980.

[4] H. B. Keller and D. S. Cohen, "Some positone problems suggested by nonlinear heat generation," Journal of Applied Mathematics and Mechanics, vol. 16, no. 12, pp. 1361-1376, 1967.

[5] A. C. Lazer and P. J. McKenna, "On a singular nonlinear elliptic boundary-value problem," Proceedings of the American Mathematical Society, vol. 111, no. 3, pp. 721-730, 1991.
[6] A. V. Lair and A. W. Shaker, "Classical and weak solutions of a singular semilinear elliptic problem," Journal of Mathematical Analysis and Applications, vol. 211, no. 2, pp. 371-385, 1997.

[7] J. Shi and M. Yao, "Positive solutions for elliptic equations with singular nonlinearity," Electronic Journal of Differential Equations, vol. 2005, no. 4, pp. 1-11, 2005.

[8] L. Boccardo and L. Orsina, "Semilinear elliptic equations with singular nonlinearities," Calculus of Variations and Partial Differential Equations, vol. 37, no. 3-4, pp. 363-380, 2010.

[9] F. Li and Z. Yang, "Existence of positive solutions of singular pLaplacian equations in a ball," Journal of Nonlinear Science and its Applications, vol. 5, no. 1, pp. 44-55, 2012.

[10] J. Carmona, P. J. Martínez-Aparicio, and J. D. Rossi, "A singular elliptic equation with natural growth in the gradient and a variable exponent," Nonlinear Differential Equations and Applications, vol. 22, no. 6, pp. 1935-1948, 2015.

[11] L. Boccardo and T. Gallouet, "Nonlinear elliptic equations with right-hand side measures," Communications in Partial Differential Equations, vol. 17, no. 3-4, pp. 641-655, 1992.

[12] M. M. Coclite and G. Palmieri, "On a singular nonlinear Dirichlet problem," Communications in Partial Differential Equations, vol. 14, no. 10, pp. 1315-1327, 1989.

[13] G. Stampacchia, "Le Problème de Dirichlet pour les équations elliptiques du second ordre à coefficients discontinus," Annales de l'Institut Fourier, vol. 15, no. 1, pp. 189-257, 1965.

[14] D. Arcoya and D. Ruiz, "The Ambrosetti-Prodi problem for the p-Laplacian operator," Communications in Partial Differential Equations, vol. 31, no. 4-6, pp. 849-865, 2006.

[15] J. L. Vázquez, "A strong maximum principle for some quasilinear elliptic equations," Applied Mathematics and Optimization, vol. 12, no. 3, pp. 191-202, 1984. 


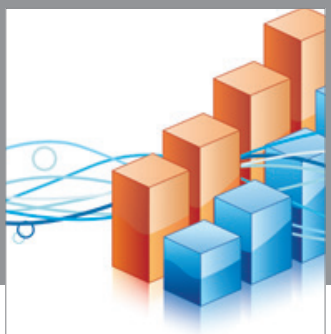

Advances in

Operations Research

vatem alat4

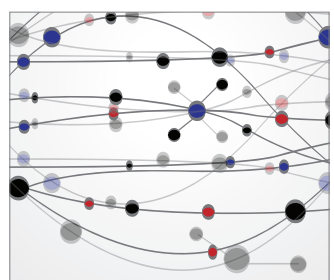

\section{The Scientific} World Journal
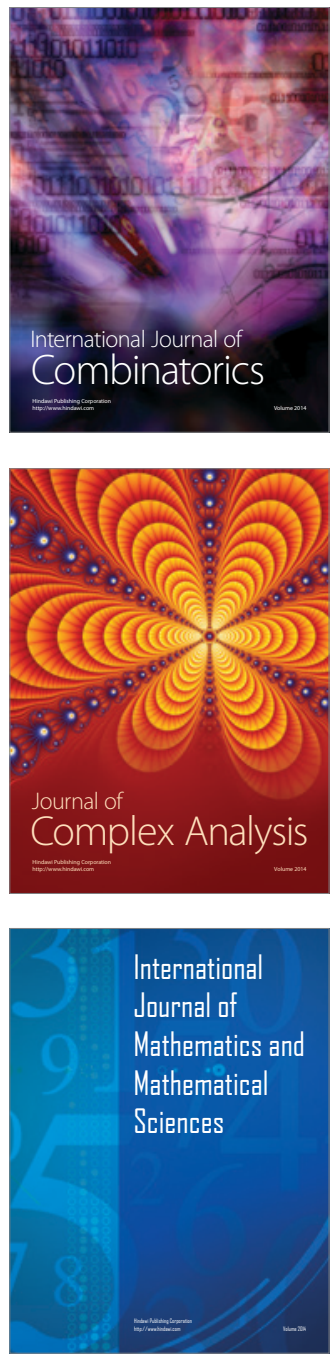
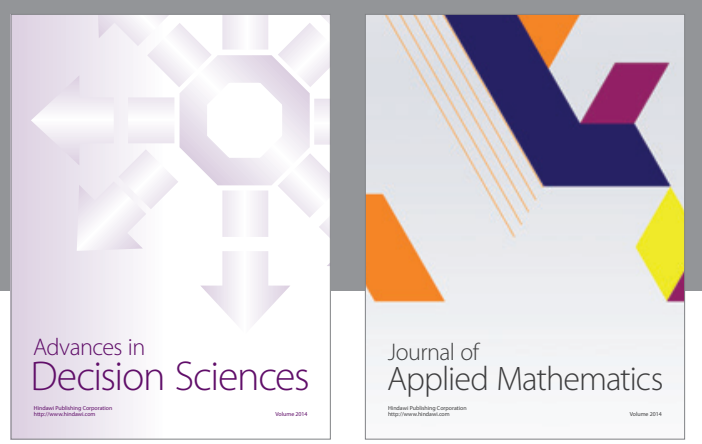

Algebra

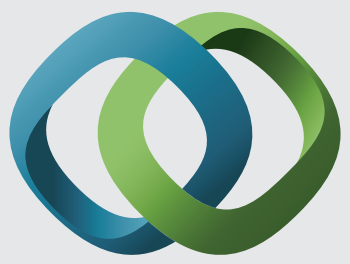

\section{Hindawi}

Submit your manuscripts at

http://www.hindawi.com
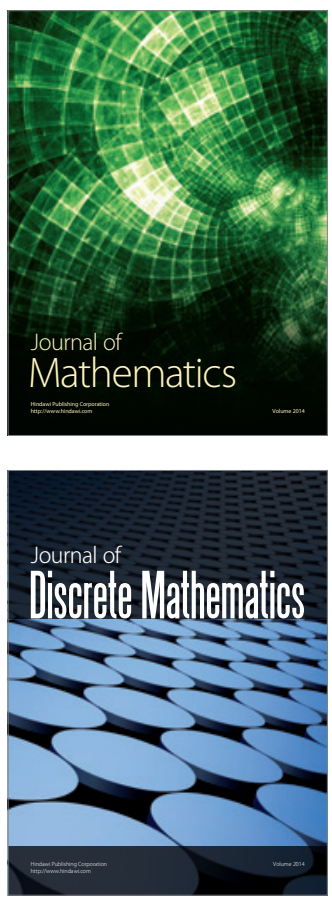

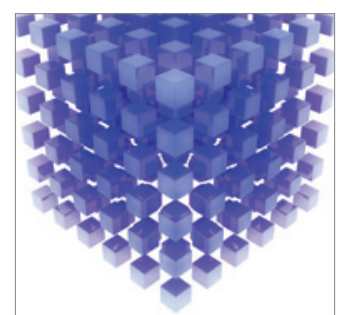

Mathematical Problems in Engineering
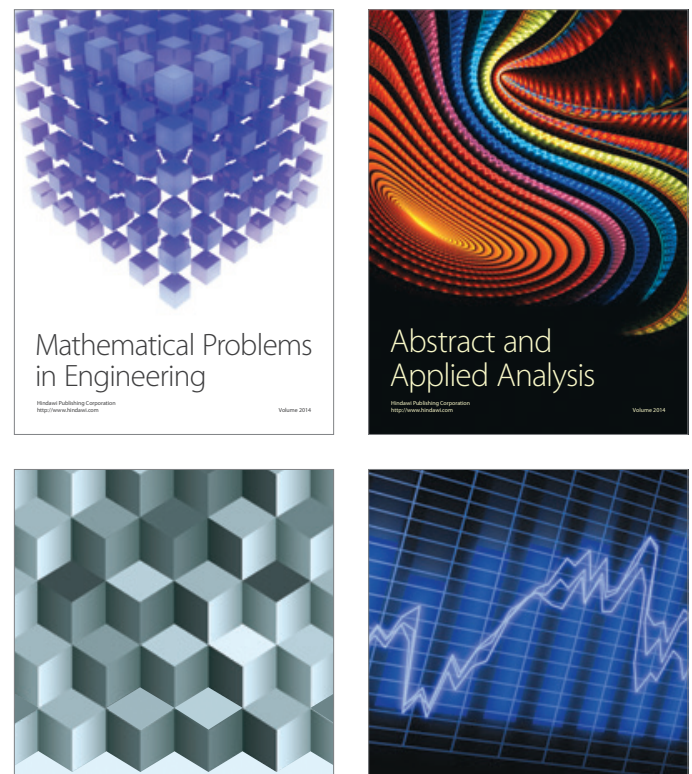

Journal of

Function Spaces

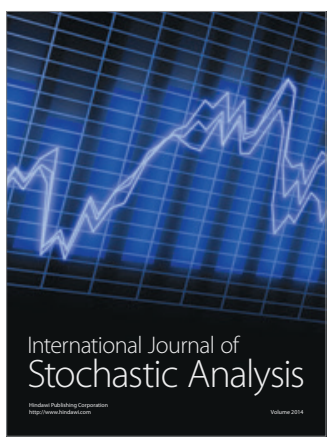

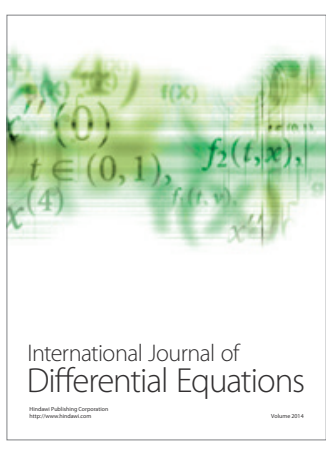
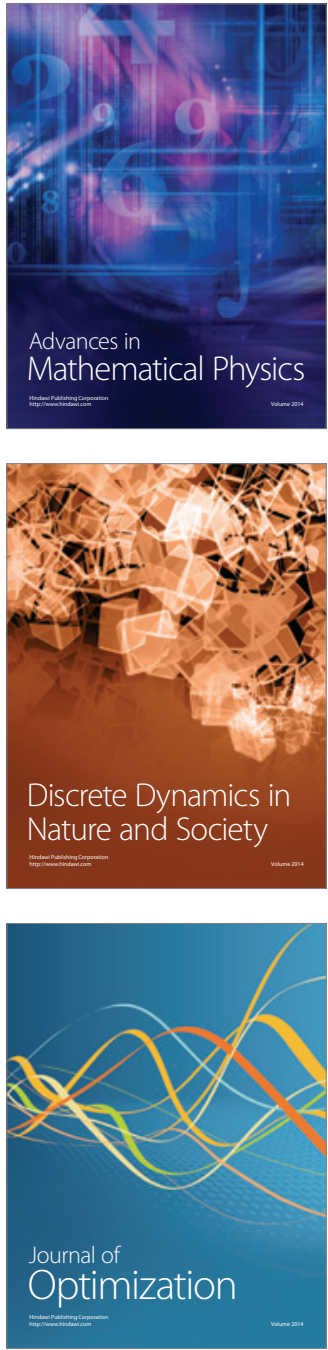